Proceedings of the Institute of Mathematics and Mechanics,

National Academy of Sciences of Azerbaijan

Volume 47, Number 1, 2021, Pages 183-196

https://doi.org/10.30546/2409-4994.47.1/183

\title{
ON A PROBLEM OF SYNTHESIS OF CONTROL OF POWER OF THE MOVING SOURCES ON HEATING OF A ROD
}

\author{
KAMIL R. AIDA-ZADE, VUGAR A. HASHIMOV, AND ARZU H. BAGIROV
}

\begin{abstract}
The problem of synthesis of control of power of the moving sources according to the given rules and trajectories under heating the rod is considered. The current values of the controls are determined depending on the values of the temperature of the rod at the points of measurement. Formulas for the components of the gradient of the objective functional are obtained with respect to the feedback parameters and the coordinates of the placement of the measurement points. The formulas can be used in the numerical solution to the test problem using first-order numerical optimization methods.
\end{abstract}

\section{Introduction}

We consider the problem of optimal synthesis of control of power of the sources under heating a rod moving on given trajectories and accordingly given laws The current values of the powers of the sources are assigned depending on the measured values of temperature of the rod at the points at which the measuring devices are set.

In recent years, due to development of computing and measuring technology, interest in the study control problems with feedback with a controlled object has increased. To control and regulate some industrial processes and technical objects special control devices were developed already in the last century using the research results of J.C. Maxwell, E.J., Routh, I.A. Vyshnegradskiy, A. Hurwitz, A.M. Lyapunov and other scientists and engineers. Later, the results of research of L.S. Pontryagin, R.E. Bellman, A.M. Letov and many other scientists made it possible to solve serious problems in rocketry, astronautics and create the systems for automatic control of various technological processes, industrial objects $[7],[8],[9],[10],[14],[15],[16],[17],[18]$.

The originality of the results of this work regarding the problems of synthesis of optimal control of systems with distributed parameters is that the synthesis of the power of control of the moving sources is carried out; for assignment the current values of powers of the sources a formula of linear dependence on the measured temperature values at the measurement points, the placements of which need to

2010 Mathematics Subject Classification. 49M05, 49K20.

Key words and phrases. rod heating, feedback control, moving sources, points of temperature measurement, feedback parameters. 
be optimized is proposed; the considered control synthesis problem is reduced to a finite-dimensional optimization problem.

A specific feature of the problem under consideration is, firstly, that the current values of the powers of moving lumped sources are distributed along the rod in some neighbourhood of their current locations; secondly, the current state of the rod is influenced not only by the instant values of the power of the sources, but also by the integral values during a certain specified time interval.

In this work we obtained formulas for the components of the gradient of the objective functional with respect to the feedback parameters and the location of the measuring points, which allow using standard numerical methods of optimization of the gradient type to solve the control synthesis problem.

The proposed approach can be used in the systems for control and regulation sources for objects described by other kinds and types of initial-boundary value problems.

\section{Formulation of the problem}

Consider the rod heating process described by the following initial-boundary value problem regarding to integral-differential equation [5]:

$$
\begin{gathered}
u_{t}(x, t)=a^{2} u_{x x}(x, t)-\lambda_{0}[u(x, t)-\theta]+\sum_{i=1}^{N_{s}} q^{i}(t) \mu_{\sigma^{\mathrm{s}}}\left(x ; z^{i}(t)\right), \\
(x, t) \in(0, l) \times(0, T], \\
u(x, 0)=b(x)=b=\mathrm{const}, x \in[0, l], \\
u_{x}(0, t)=\lambda[u(0, t)-\theta], u_{x}(l, t)=\lambda[u(l, t)-\theta], t \in(0, T],
\end{gathered}
$$

Here: $u(x, t)$ is the temperature of the rod at a point $x \in[0, l]$ at the moment $t$; $a, \lambda, \lambda_{0}$ are given parameters of the heating process; $\theta$ is ambient temperature.

Continuously differentiable with respect to $x$ function $\mu_{\sigma^{\mathrm{s}}}(x ; \hat{z}), \hat{z} \in\left[\sigma^{\mathrm{s}}, l-\sigma^{\mathrm{s}}\right]$ for given positive parameter $\sigma^{\mathrm{s}}$, determine the distribution of the instant value of the $q(t)$ power of the source at time $t$, lumped at the point $\hat{z}$, in a domain $\left(\hat{z}-\sigma^{\mathrm{s}}, \hat{z}+\sigma^{\mathrm{s}}\right)$ :

$$
\mu_{\sigma^{\mathrm{s}}}(x ; \hat{z}) \begin{cases}\geq 0, & \text { if } x \in\left(\hat{z}-\sigma^{\mathrm{s}}, \hat{z}+\sigma^{\mathrm{s}}\right), \\ =0, & \text { if } x \notin\left(\hat{z}-\sigma^{\mathrm{s}}, \hat{z}+\sigma^{\mathrm{s}}\right) .\end{cases}
$$

Moreover, these functions must satisfy the conditions:

$$
\int_{\hat{z}-\sigma^{\mathrm{s}}}^{\hat{z}+\sigma^{\mathrm{s}}} \mu_{\sigma^{\mathrm{s}}}(x ; \hat{z}) d x=1 .
$$

It is clear that for $\sigma^{\mathrm{s}}$ tending to 0 the function $\mu_{\sigma^{\mathrm{s}}}(x ; 0)$ tends to the $\delta$-function of Dirac [12]. But the problem considered in this work is closer to real applications.

The heating process is carried out by $N_{s}$ sources moving along given trajectories, powers $q=q(t)=\left(q^{1}(t), \ldots, q^{N_{s}}(t)\right)$, which are piece-wise continuous controls, satisfy the constraints:

$$
q^{i}(t) \in Q^{i}=\left[\underline{q^{i}}, \overline{q^{i}}\right], \quad i=1, \ldots, N_{s}, \quad t \in[0, T),
$$


where values $q^{i}, \overline{q^{i}}, i=1, \ldots, N_{s}$ are given. Continuous functions $z^{i}(t) \in$ $\left[\sigma^{\mathrm{s}}, l-\sigma^{\mathrm{s}}\right]$ are given, they determine the position of $i$-th power source at the moment $t$ on the rod, $i=1, \ldots, N_{s}$.

We consider the initial temperature in (2.2) at all points of the rod to be the same, but not exactly specified, and belonging to a given set $B \subset \mathrm{R}$ with a known density function $\rho_{B}(b)$ :

$$
\rho_{B}(b) \geq 0, b \in B, \int_{B} \rho_{B}(b) d b=1 .
$$

Ambient temperature $\theta$ is constant, and its values belong to the set $\Theta \subset \mathrm{R}$ with a given density function $\rho_{\Theta}(\theta)$ with properties

$$
\rho_{\Theta}(\theta) \geq 0, \theta \in \Theta, \int_{\Theta} \rho_{\Theta}(\theta) d \theta=1
$$

The formulated problem is to determine the values of the sources power $q=$ $q(t)=\left(q^{1}(t), \ldots, q^{N_{s}}(t)\right) \in Q$ which minimize the following objective functional:

$$
\begin{gathered}
J_{T}(q)=\int_{B} \int_{\Theta} I_{T}(q ; b, \theta) \rho_{B}(b) \rho_{\Theta}(\theta) d \theta d b, \\
I_{T}(q ; b, \theta)=\int_{0}^{l} \zeta(x)[u(x, T)-U(x)]^{2} d x+\varepsilon\|q(t)-\hat{q}(t)\|_{L_{2}^{N_{s}}[0, T]}^{2} .
\end{gathered}
$$

Here: function $u(x, t)=u(x, t ; q, b, \theta)$ is the solution to the initial-boundary value problem $(2.1)-(2.3)$ at $u(x, 0)=b$, ambient temperature $\theta$, values of sources power $q(t)$; function $U(x), x \in[0, l]$ is the desired state of the rod at $t=T$; $\zeta(x) \geq 0, x \in[0, l]$ is the weight function; $\varepsilon, \hat{q}(t)$ are regularization parameters of the objective functional of the problem [19].

Let at the given $N_{c}$ points of the $\operatorname{rod} \xi^{j} \in\left[\sigma^{\mathrm{c}}, l-\sigma^{\mathrm{c}}\right], j=1, \ldots, N_{c}$ temperature measurements in the process of its heating are carried out:

$$
u_{j}(t)=\int_{\xi^{j}-\sigma^{\mathrm{c}}}^{\xi^{j}+\sigma^{\mathrm{c}}} u(x, t) \nu_{\sigma^{\mathrm{c}}}\left(x ; \xi^{j}\right) d x, j=1, \ldots, N_{c}, t \in[0, T],
$$

continuously in time or at given times $t_{k} \in[0, T]$ :

$$
u_{j}^{k}=\int_{\xi^{j}-\sigma^{\mathrm{c}}}^{\xi^{j}-\sigma^{\mathrm{c}}} u\left(x, t_{k}\right) \nu_{\sigma^{\mathrm{c}}}\left(x ; \xi^{j}\right) d x, j=1, \ldots, N_{c}, k=0, \ldots, N_{t} .
$$

The relations (2.7) and (2.8) indicate that the assignment of the measured current state at the point $\xi^{j}$ affects the states at the points of some of its neighborhood: $\left(\xi^{j}-\sigma^{\mathrm{c}}, \xi^{j}+\sigma^{\mathrm{c}}\right)$ with weight function $\nu_{\sigma^{\mathrm{c}}}\left(x ; \xi^{j}\right), j=1 \ldots, N_{c}$.

The weight function $\nu_{\sigma^{c}}\left(\sigma^{\mathrm{c}} ; \hat{\xi}\right)$ has the following properties:

$$
\nu_{\sigma^{\mathrm{c}}}(x ; \hat{\xi}) \begin{cases}\geq 0, & \text { if } x \in\left(\hat{\xi}-\sigma^{\mathrm{c}}, \hat{\xi}+\sigma^{\mathrm{c}}\right), \\ =0, & \text { if } x \notin\left(\hat{\xi}-\sigma^{\mathrm{c}}, \hat{\xi}+\sigma^{\mathrm{c}}\right),\end{cases}
$$




$$
\int_{\hat{\xi}-\sigma^{\mathrm{c}}}^{\hat{\xi}+\sigma^{\mathrm{c}}} \nu_{\sigma^{\mathrm{c}}}(x ; \hat{\xi}) d x=1 .
$$

Based on the obtained measurement the power values $q^{i}(t), i=1, \ldots, N_{s}$ are determined using the following relationship:

$$
q^{i}(t)=\sum_{j=1}^{N_{c}} \alpha_{i}^{j}\left[\int_{\xi^{j}-\sigma^{c}}^{\xi^{j}+\sigma^{c}} u(x, t) \nu_{\sigma^{c}}\left(x ; \xi^{j}\right) d x-\hat{\omega}_{i}^{j}\right], i=1, \ldots, N_{s}, t \in[0, T),
$$

where $\alpha_{i}^{j}, \hat{\omega}_{i}^{j}, \xi^{j}$ are coefficients of feedback, $i=1, \ldots, N_{s}, j=1, \ldots, N_{c}$ [4], [11].

The value in brackets is equal to the deviation of the temperature at the $j$-th measuring point from the value $\hat{\omega}_{i}^{j}$ that is nominal relative to the $i$-th source for the $j$-th measuring point, $\alpha_{i}^{j}$ are the coefficients of the amplification.

Formula (2.9) can be written as follows:

$$
q^{i}(t)=\sum_{j=1}^{N_{c}} \alpha_{i}^{j} \int_{\xi^{j}-\sigma^{\mathrm{c}}}^{\xi^{j}+\sigma^{\mathrm{c}}} u(x, t) \nu_{\sigma^{\mathrm{c}}}\left(x ; \xi^{j}\right) d x-\sum_{j=1}^{N_{c}} \alpha_{i}^{j} \hat{\omega}_{i}^{j}, i=1, \ldots, N_{s}, t \in[0, T),
$$

and after notation

$$
\sum_{j=1}^{N_{c}} \alpha_{i}^{j} \hat{\omega}_{i}^{j}=\omega^{i}, i=1, \ldots, N_{s}
$$

we have:

$$
q^{i}(t)=\sum_{j=1}^{N_{c}} \alpha_{i}^{j} \int_{\xi^{j}-\sigma^{c}}^{\xi^{j}+\sigma^{\mathrm{c}}} u(x, t) \nu_{\sigma^{\mathrm{c}}}\left(x ; \xi^{j}\right) d x-\omega^{i}, i=1, \ldots, N_{s}, t \in[0, T) .
$$

The number of feedback coefficients (parameters) in formulas (2.9) and (2.10) is $n=N_{s} N_{c}+N_{s}+N_{c}$. Further for the control influence $q^{i}(t), i=1, \ldots, N_{s}$ we will use the dependency (2.10).

In the case of measurements (2.8) that are discrete in time, we use the formula:

$$
\begin{gathered}
q^{i}(t)=\sum_{j=1}^{N_{c}} \alpha_{i}^{j} \int_{\xi^{j}-\sigma^{c}}^{\xi^{j}+\sigma^{\mathrm{c}}} u\left(x, t_{k}\right) \nu_{\sigma^{\mathrm{c}}}\left(x ; \xi^{j}\right) d x-\omega^{i}, i=1, \ldots, N_{s}, t \in\left[t_{k}, t_{k+1}\right), \\
k=0, \ldots, N_{t}, t_{0}=0, t_{N_{t}+1}=T .
\end{gathered}
$$

Since the function $u(x, t)$ is the solution of problem (2.1)-(2.3) is continuous by $t$ at $t \in[0, T]$, then the power $q(t)$, obtained from formula (2.10), in measurements (2.7) are continuous functions. If measurements are taken discretely in time, then the power functions $q^{i}(t), i=1, \ldots, N_{s}$, according to formula (2.11) are piece-wise continuous for $t \in[0, T]$ and are continuous at $t \in\left[t_{k}, t_{k+1}\right), k=0, \ldots, N_{t}$.

Using formula (2.9) in the case of continuous feedback (2.10) in equation (2.1), we get:

$$
u_{t}(x, t)=a^{2} u_{x x}(x, t)-\lambda_{0}[u(x, t)-\theta]+
$$




$$
\begin{gathered}
+\sum_{i=1}^{N_{s}}\left[\sum_{j=1}^{N_{c}} \alpha_{i}^{j} \int_{\xi^{j}-\sigma^{\mathrm{c}}}^{\xi^{j}+\sigma^{\mathrm{c}}} u(\gamma, t) \nu_{\sigma^{\mathrm{c}}}\left(\gamma ; \xi^{j}\right) d \gamma-\omega^{i}\right] \mu_{\sigma^{\mathrm{s}}}\left(x ; z^{i}(t)\right), \\
x \in(0, l), t \in(0, T] .
\end{gathered}
$$

If the feedback is discrete in time, substituting (2.11) into (2.1), for the heating process we obtain the following equations, defined on time half-intervals $t \in$ $\left[t_{k}, t_{k+1}\right), k=0, \ldots, N_{t}$ :

$$
\begin{gathered}
u_{t}(x, t)=a^{2} u_{x x}(x, t)-\lambda_{0}[u(x, t)-\theta]+ \\
+\sum_{i=1}^{N_{s}}\left[\sum_{j=1}^{N_{c}} \alpha_{i}^{j} \int_{\substack{\xi^{j}-\sigma^{c} \\
\xi^{j}+\sigma^{c}}} u\left(\gamma, t_{k}\right) \nu_{\sigma^{\mathrm{c}}}\left(\gamma ; \xi^{j}\right) d \gamma-\omega^{i}\right] \mu_{\sigma^{\mathrm{s}}}\left(x ; z^{i}(t)\right), \\
x \in(0, l), t \in\left[t_{k}, t_{k+1}\right), k=0, \ldots, N_{t} .
\end{gathered}
$$

In this case, the conditions for the continuity of the process as a whole are satisfied:

$$
u\left(x, t_{k}^{-}\right)=u\left(x, t_{k}\right)=u\left(x, t_{k}^{+}\right), x \in[0, l], k=1, \ldots, N_{t} .
$$

Equations (2.12) and (2.13) are integro-differential or integrally loaded. The corresponding initial-boundary value problems have been studied in many works (the bibliography can be found in [13]). By discrete approximation of the integral in the equation is reduced to a point-loaded equation. The study of such equations was carried out in $[1],[2],[3],[6],[13]$.

The problem considered below is to determine the feedback coefficients $\alpha=$ $\left(\alpha_{i}^{j}\right), \omega=\left(\omega^{i}\right), \xi=\left(\xi^{j}\right), j=1, \ldots, N_{c}, i=1, \ldots, N_{s}$ under constraints $(2.4)$ that provide the objective functional with a minimum value. For the parameters optimized in the problem, we introduce the notation $y=(\alpha, \omega, \xi) \in \mathrm{R}^{n}$, and functional $(2.5),(2.6)$ of the considered problem can be written as follows:

$$
\begin{gathered}
J_{T}(y)=\int_{B} \int_{\Theta} I_{T}(y ; b, \theta) \rho_{B}(b) \rho_{\Theta}(\theta) d \theta d b, \\
I_{T}(y ; b, \theta)=\int_{0}^{l} \zeta(x)[u(x, T)-U(x)]^{2} d x+\varepsilon\|y-\hat{y}\|_{\mathrm{R}^{n}}^{2} .
\end{gathered}
$$

Here: $u(x, t)=u(x, t ; y, b, \theta)$ solution of the initial-boundary value problem with respect to equation $(2.12),(2.13),(2.2),(2.3)$ for the given parameters $y=(\alpha, \omega, \xi)$, with the initial condition $u(x, 0)=b$ and ambient temperature $\theta$.

Constraints (2.4) on the power of sources with continuous feedback (2.10) will become joint constraints on the parameters $y$ and the phase state temperature at the measuring points $\xi^{j}$ in integral form, $j=1, \ldots, N_{c}$.

$$
\begin{gathered}
\underline{q^{i} \leq q^{i}(t ; y)=} \sum_{j=1}^{N_{c}} \alpha_{i}^{j} \int_{\xi^{j}-\sigma^{\mathrm{c}}}^{\xi^{j}+\sigma^{\mathrm{c}}} u(x, t) \nu_{\sigma^{\mathrm{c}}}\left(x ; \xi^{j}\right) d x-\omega^{i} \leq \overline{q^{i}}, \\
t \in[0, T), i=1, \ldots, N_{s} .
\end{gathered}
$$


We can write these restrictions in the equivalent form

$$
\begin{gathered}
g^{i}(t ; y)=\left|g_{0}^{i}(t ; y)\right|-\frac{\overline{q^{i}}-\underline{q^{i}}}{2} \leq 0, t \in[0, T), i=1, \ldots, N_{s}, \\
g_{0}^{i}(t ; y)=\frac{\overline{q^{i}}+\underline{q^{i}}}{2}-q^{i}(t ; y) .
\end{gathered}
$$

The resulting problem $(2.12),(2.2)-(2.4),(2.14),(2.15)$ belongs to the class of problems of control synthesis for systems with distributed parameters, in which the sought for is the finite-dimensional vector $y \in \mathrm{R}^{n}$. The specifics of the problem are: integro-differential equations describing the process under study; the dependence of the values of the objective functional on the set of solutions of the initial-boundary value problem, since the initial condition of the rod and the ambient temperature can take values from the sets $B$ and $\Theta$. Note that the dimension of the resulting finite-dimensional optimization problem to which the original problem is reduced is determined by the double product of the number of sources and sampling points. This dimension can be considered acceptable, given the current state of computer technology and numerical optimization methods.

\section{Approach and formulas for solving the problem}

First of all, note the convexity in $q(t)$ the source optimal control problem (2.1)-(2.6) (without feedback). It is clear that the functional of the control problem (2.14), (2.15) with both continuous (2.10) and discrete (2.11) feedback is not convex with respect to the optimized feedback parameters $y$. Note also the non-convexity of the permissible parameter range $y$, defined by formulas (2.16). This follows the non-linearity of the dependence of the solution to the initialboundary value problem $u(x, t)$ from parameters $y=(\alpha, \omega, \xi)$. The formulas for the gradients of the functional given below in the theorems can be useful for the numerical solution of the problem of determining the locally optimal soughtfor feedback parameters or for local clarification of their values specified by the expert. In general, to optimize the feedback parameters, global optimization methods can be used in combination with local methods of conditional gradient optimization.

To solve the resulting finite-dimensional parametric optimal control problem (2.1)-(2.6), we apply the penalty function method to take into account constraints (2.17) [19]. Taking into account, as indicated above, the multi-extremality of the problem in terms of feedback parameters for both continuous and discrete feedback, the solution of the problem can be solved using the method of penalty functions with different initial search points relative to the vector of parameters $y$.

The external penalty functional with respect to functional $(2.14),(2.15)$ is written in the form:

$$
J_{T, \mathcal{R}}(y)=\int_{B} \int_{\Theta} I_{T}(y ; b, \theta) \rho_{B}(b) \rho_{\Theta}(\theta) d \theta d b,
$$




$$
\begin{gathered}
I_{T, \mathcal{R}}(y ; b, \theta)=\int_{0}^{l} \zeta(x)[u(x, T)-U(x)]^{2} d x+\varepsilon\|y-\hat{y}\|_{\mathrm{R}^{n}}^{2}+\mathcal{R} G_{q}(y), \\
G_{q}(y)=\sum_{i=1}^{N_{s}} \int_{0}^{T}\left[g_{+}^{i}(t ; y)\right]^{2} d t .
\end{gathered}
$$

Here the coefficient $\mathcal{R}$ tends to infinity, and the function $g_{+}^{i}(t ; y)=0$, if $g^{i}(t ; y) \leq$ 0 , and $g_{+}^{i}(t ; y)=g^{i}(t ; y)$, if $g^{i}(t ; y)>0$.

Theorem 3.1. With continuous feedback (2.10), the functional $J_{T, \mathcal{R}}(y)$ of problem (2.12), (2.2), (2.3), (2.4), (3.1), (3.2) for each value of the coefficient $\mathcal{R}$ differentiable by $y=(\alpha, \omega, \xi)$, and the components of its gradient have the formulas

$$
\begin{aligned}
\frac{\partial J_{T, \mathcal{R}}(y)}{\partial \alpha_{i}^{j}} & =\int_{B} \int_{\Theta}\left\{-\int_{0}^{T}\left[\int_{z^{i}(t)-\sigma^{\mathrm{s}}}^{z^{i}(t)+\sigma^{\mathrm{s}}} \psi(x, t) \mu_{\sigma^{\mathrm{s}}}\left(x ; z^{i}(t)\right) d x+\right.\right. \\
& \left.+2 \mathcal{R} g_{+}^{i}(t ; y) \operatorname{sgn}\left(g_{0}^{i}(t ; y)\right)\right]\left[\int_{\xi^{j}-\sigma^{\mathrm{c}}}^{\xi^{j}+\sigma^{\mathrm{c}}} u(\gamma, t) \nu_{\sigma^{\mathrm{c}}}\left(\gamma ; \xi^{j}\right) d \gamma\right] d t+ \\
& \left.+2 \varepsilon\left(\alpha_{i}^{j}-\hat{\alpha}_{i}^{j}\right)\right\} \rho_{B}(b) \rho_{\Theta}(\theta) d \theta d b,
\end{aligned}
$$

$$
\begin{aligned}
\frac{\partial J_{T, \mathcal{R}}(y)}{\partial \omega^{i}} & =\iint_{B} \int_{\Theta}\left\{\int _ { 0 } ^ { T } \left[\int_{z^{i}(t)-\sigma^{\mathrm{s}}}^{z^{i}(t)+\sigma^{\mathrm{s}}} \psi(x, t) \mu_{\sigma^{\mathrm{s}}}\left(x ; z^{i}(t)\right) d x+\right.\right. \\
& \left.\left.+2 \mathcal{R} g_{+}^{i}(t ; y) \operatorname{sgn}\left(g_{0}^{i}(t ; y)\right)\right] d t+2 \varepsilon\left(\omega^{i}-\hat{\omega}^{i}\right)\right\} \rho_{B}(b) \rho_{\Theta}(\theta) d \theta d b,
\end{aligned}
$$

$$
\begin{aligned}
\frac{\partial J_{T, \mathcal{R}}(y)}{\partial \xi^{j}} & =\int_{B} \int_{\Theta}\left\{-\sum_{i=1}^{N_{s}} \int_{0}^{T}\left[\int_{z^{i}(t)-\sigma^{\mathrm{s}}}^{z^{i}(t)+\sigma^{\mathrm{s}}} \psi(x, t) \mu_{\sigma^{\mathrm{s}}}\left(x ; z^{i}(t)\right) d x+\right.\right. \\
+ & \left.2 \mathcal{R} g_{+}^{i}(t ; y) \operatorname{sgn}\left(g_{0}^{i}(t ; y)\right)\right]\left[\int_{\xi^{j}-\sigma^{\mathrm{c}}}^{\xi^{j}+\sigma^{\mathrm{c}}} u_{\gamma}(\gamma, t) \nu_{\sigma^{\mathrm{c}}}\left(\gamma ; \xi^{j}\right) d \gamma\right] d t+ \\
& \left.+2 \varepsilon\left(\xi^{j}-\hat{\xi}^{j}\right)\right\} \rho_{B}(b) \rho_{\Theta}(\theta) d \theta d b,
\end{aligned}
$$


$i=1, \ldots, N_{s}, j=1, \ldots, N_{c}$. Here the function $\psi(x, t)=\psi(x, t ; y, b, \theta, \mathcal{R})$ is the solution for the following conjugate initial-boundary value problem:

$$
\begin{gathered}
\psi_{t}(x, t)=-a^{2} \psi_{x x}(x, t)+\lambda_{0} \psi(x, t)-\sum_{j=1}^{N_{c}} \nu_{\sigma^{\mathrm{c}}}\left(x ; \xi^{j}\right) . \\
\sum_{i=1}^{N_{s}}\left\{\alpha_{i}^{j} \int_{z^{i}(t)-\sigma^{\mathrm{s}}}^{z^{i}(t)+\sigma^{\mathrm{s}}} \psi(\gamma, t) \mu_{\sigma^{\mathrm{s}}}\left(\gamma ; z^{i}(t)\right) d \gamma+2 \mathcal{R} g_{+}^{i}(t ; y) \operatorname{sgn}\left(g_{0}^{i}(t ; y)\right)\right\} d t \\
x \in(0, l), t \in[0, T) \\
\psi(x, T)=-2 \zeta(x)[u(x, T)-U(x)], x \in[0, l] \\
\psi_{x}(0, t)=\lambda \psi(0, t), \psi_{x}(l, t)=-\lambda \psi(l, t), t \in[0, T)
\end{gathered}
$$

Proof. From the independence of the initial temperature of the rod and the temperature of the external environment from the vector of its parameters, it follows:

$$
\begin{gathered}
\operatorname{grad}_{y} J_{T, \mathcal{R}}(y)=\operatorname{grad} \int_{B} \int_{\Theta} I_{T, \mathcal{R}}(y ; b, \theta) \rho_{\Theta}(\theta) \rho_{B}(b) d \theta d b= \\
=\int_{B} \int_{\Theta} \operatorname{grad}_{y} I_{T, \mathcal{R}}(y ; b, \theta) \rho_{\Theta}(\theta) \rho_{B}(b) d \theta d b .
\end{gathered}
$$

Taking this into account, further we will deal with the differentiability of functional (2.15) at given temperatures $b$ and $\theta$.

In equation (2.12) we use the notation:

$$
F(x, t ; u, y)=\sum_{i=1}^{N_{s}}\left[\sum_{j=1}^{N_{c}} \alpha_{i}^{j} \int_{\xi^{j}-\sigma^{c}}^{\xi^{j}+\sigma^{\mathrm{c}}} u(\gamma, t) \nu_{\sigma^{\mathrm{c}}}\left(\gamma ; \xi^{j}\right) d \gamma-\omega^{i}\right] \mu_{\sigma^{\mathrm{s}}}\left(x ; z^{i}(t)\right),
$$

and equation (2.12) is written as follows:

$$
u_{t}(x, t)=a^{2} u_{x x}(x, t)-\lambda_{0}[u(x, t)-\theta]+F(x, t ; u, y) .
$$

Further, we will apply the increment method for independent variables $y$. Suppose the parameters $y=(\alpha, \omega, \xi)$ got an increment $\Delta y$, let denote $y_{1}=y+\Delta y=$ $(\alpha+\Delta \alpha, \omega+\Delta \omega, \xi+\Delta \xi)$. It is clear that the influence $F(x, t ; u, y)$ will be increased:

$$
\Delta F(x, t ; u, y)=F\left(x, t ; u, y_{1}\right)-F(x, t ; u, y)
$$

and phase variable

$$
\Delta u(x, t ; y)=u\left(x, t ; y_{1}\right)-u(x, t ; y) .
$$

Then the function $\Delta u(x, t ; y)$ is the solution to the problem:

$$
\begin{gathered}
\Delta u_{t}(x, t)=a^{2} \Delta u_{x x}(x, t)-\lambda_{0} \Delta u(x, t)+\Delta F(x, t ; u, y), \\
x \in(0, l), t \in(0, T], \\
\Delta u(x, 0)=0, x \in[0, l], \\
\Delta u_{x}(0, t)=\lambda \Delta u(0, t), \Delta u_{x}(l, t)=-\lambda \Delta u(l, t), t \in(0, T],
\end{gathered}
$$


For the first term of the increment of functional (3.2):

$$
\Delta I_{T, \mathcal{R}}(y ; b, \theta)=\Delta I_{T}(y ; b, \theta)+\mathcal{R} \Delta G_{q}(y),
$$

taking into account (3.13)-(3.15), we have:

$$
\begin{gathered}
\Delta I_{T}(y ; b, \theta)=I_{T}\left(y_{1} ; b, \theta\right)-I_{T}(y ; b, \theta)= \\
=\int_{0}^{l} 2 \zeta(x)[u(x, T)-U(x)] \Delta u(x, T) d x+2 \varepsilon\langle y-\hat{y}, \Delta y\rangle .
\end{gathered}
$$

Let us multiply both sides of equation (3.13) by the still arbitrary function $\psi(x, t)$, integrate by $x, x \in[0, l]$ and by $t, t \in[0, T]$, and move all terms to the left. Adding the obtained relation to (3.17), we obtain

$$
\begin{aligned}
& \Delta I_{T}(y ; b, \theta)=\int_{0}^{l} 2 \zeta(x)[u(x, T)-U(x)] \Delta u(x, T) d x+2 \varepsilon\langle y-\hat{y}, \Delta y\rangle+\quad(3.1 \\
& +\int_{0}^{T} \int_{0}^{l} \psi(x, t)\left(\Delta u_{t}(x, t)-a^{2} \Delta u_{x x}(x, t)+\lambda_{0} \Delta u(x, t)-\Delta F(x, t ; u, y)\right) d x d t .
\end{aligned}
$$

Integrating by parts we get:

$$
\begin{gathered}
\Delta I_{T}(y ; b, \theta)=\int_{0}^{l} 2 \zeta(x)[u(x, T)-U(x)] \Delta u(x, T) d x+2 \varepsilon\langle y-\hat{y}, \Delta y\rangle+\quad(3.19) \\
+\int_{0}^{l} \psi(x, T) \Delta u(x, T) d x-\int_{0}^{l} \psi(x, 0) \Delta u(x, 0) d x-a^{2} \int_{0}^{T} \psi(l, t) \Delta u_{x}(l, t) d t+ \\
+a^{2} \int_{0}^{T} \psi(0, t) \Delta u_{x}(0, t) d t+a^{2} \int_{0}^{T} \psi_{x}(l, t) \Delta u(l, t) d t-a^{2} \int_{0}^{T} \psi_{x}(0, t) \Delta u(0, t) d t- \\
-\int_{0}^{T} \int_{0}^{l}\left(\psi_{t}(x, t)+a^{2} \psi_{x x}(x, t)-\lambda_{0} \psi(x, t)\right) \Delta u(x, t) d x d t- \\
-\int_{0}^{T} \int_{0}^{l} \psi(x, t) \Delta F(x, t ; u, y) d x d t .
\end{gathered}
$$

Bracket $\langle\cdot, \cdot\rangle$ denotes scalar product operation.

For the last term (3.19), taking into account (3.9), (3.11), after transformations we obtain:

$$
\int_{0}^{T} \int_{0}^{l} \psi(x, t) \Delta F(x, t ; u, y) d x d t=\int_{0}^{T} \int_{0}^{l} \psi(x, t)\left[F\left(x, t ; u, y_{1}\right)-F(x, t ; u, y)\right] d x d t=
$$




$$
\begin{aligned}
& =\sum_{i=1}^{N_{s}} \sum_{j=1}^{N_{c}} \Delta \alpha_{i}^{j} \int_{0}^{T} \int_{z^{i}(t)-\sigma^{\mathrm{s}}}^{z^{i}(t)+\sigma^{\mathrm{s}}} \psi(x, t) \mu_{\sigma^{\mathrm{s}}}\left(x ; z^{i}(t)\right) d x \int_{\xi^{j}-\sigma^{\mathrm{c}}}^{\xi^{j}+\sigma^{\mathrm{c}}} u(\gamma, t) \nu_{\sigma^{\mathrm{c}}}\left(\gamma ; \xi^{j}\right) d \gamma d t- \\
& -\sum_{i=1}^{N_{s}} \Delta \omega^{i} \int_{0}^{T} \int_{z^{i}(t)-\sigma^{\mathrm{s}}}^{z^{i}(t)+\sigma^{\mathrm{s}}} \psi(x, t) \mu_{\sigma^{\mathrm{s}}}\left(x ; z^{i}(t)\right) d x d t+ \\
& +\sum_{j=1}^{N_{c}} \Delta \xi^{j} \sum_{i=1}^{N_{s}} \alpha_{i}^{j} \int_{0}^{T} \int_{z^{i}(t)-\sigma^{\mathrm{s}}}^{z^{i}(t)+\sigma^{\mathrm{s}}} \psi(x, t) \mu_{\sigma^{\mathrm{s}}}\left(x ; z^{i}(t)\right) d x \int_{\xi^{j}-\sigma^{\mathrm{c}}}^{\xi^{j}+\sigma^{\mathrm{c}}} u_{\gamma}(\gamma, t) \mu_{\sigma^{\mathrm{c}}}\left(\gamma ; \xi^{j}\right) d \gamma d t+ \\
& +\sum_{j=1}^{N_{c}} \sum_{i=1}^{N_{s}} \alpha_{i}^{j} \int_{0}^{T} \int_{z^{i}(t)-\sigma^{\mathrm{s}}}^{z^{i}(t)+\sigma^{\mathrm{s}}} \psi(x, t) \mu_{\sigma^{\mathrm{s}}}\left(x ; z^{i}(t)\right) d x \int_{\xi^{j}-\sigma^{\mathrm{c}}}^{\xi^{j}+\sigma^{\mathrm{c}}} \Delta u(\gamma, t) \nu_{\sigma^{\mathrm{c}}}\left(\gamma ; \xi^{j}\right) d \gamma d t .
\end{aligned}
$$

For the second term in formula (3.16), after simple transformations, we have:

$$
\begin{gathered}
\Delta G_{q}(y)=G_{q}\left(y_{1}\right)-G_{q}(y)=\sum_{i=1}^{N_{s}} \int_{0}^{T}\left\{\left[g_{+}^{i}\left(t ; y_{1}\right)\right]^{2}-\left[g_{+}^{i}(t ; y)\right]^{2}\right\} d t= \\
=\sum_{i=1}^{N_{s}} \int_{0}^{T}\left\{\left[\left|g_{0}^{i}\left(t ; y_{1}\right)\right|-\frac{\overline{q^{i}}-\underline{q^{i}}}{2}\right]^{2}-\left[\left|g_{0}^{i}(t ; y)\right|-\frac{\overline{q^{i}}-\underline{q^{i}}}{2}\right]^{2}\right\} d t= \\
=-2 \sum_{i=1}^{N_{s}} \sum_{j=1}^{N_{c}} \Delta \alpha_{i}^{j} \int_{0}^{T} g_{+}^{i}(t ; y) \operatorname{sgn}\left(g_{0}^{i}(t ; y)\right) \int_{\xi^{j}-\sigma^{\mathrm{c}}}^{\xi^{j}+\sigma^{\mathrm{c}}} u(\gamma, t) \nu_{\sigma^{\mathrm{c}}}\left(\gamma ; \xi^{j}\right) d \gamma d t+ \\
+2 \sum_{i=1}^{N_{s}} \Delta \omega^{i} \int_{0}^{T} g_{+}^{i}(t ; y) \operatorname{sgn}\left(g_{0}^{i}(t ; y)\right) d t- \\
-2 \sum_{j=1}^{N_{c}} \Delta \xi^{j} \sum_{i=1}^{N_{s}} \alpha_{i}^{j} \int_{0}^{T} g_{+}^{i}(t ; y) \operatorname{sgn}\left(g_{0}^{i}(t ; y)\right) \int_{\xi^{j}+\sigma^{\mathrm{c}}}^{\xi^{j}-\sigma^{\mathrm{c}}} u_{\gamma}(\gamma, t) \nu_{\sigma^{\mathrm{c}}}\left(\gamma ; \xi^{j}\right) d \gamma d t- \\
-2 \sum_{j=1}^{N_{c}} \sum_{i=1}^{N_{s}} \alpha_{i}^{j} \int_{0}^{T} g_{+}^{i}(t ; y) \operatorname{sgn}\left(g_{0}^{i}(t ; y)\right) \int_{\xi^{j}-\sigma^{\mathrm{c}}}^{\int^{c}} \Delta u(\gamma, t) \nu_{\sigma^{\mathrm{c}}}\left(\gamma ; \xi^{j}\right) d \gamma d t .
\end{gathered}
$$

It's clear that:

$$
\begin{array}{r}
\Delta I_{T, \mathcal{R}}(y ; b, \theta)=\int_{0}^{l} 2 \zeta(x)[u(x, T)-U(x)] \Delta u(x, T) d x+ \\
+\int_{0}^{l} \psi(x, T) \Delta u(x, T) d x-\int_{0}^{l} \psi(x, 0) \Delta u(x, 0) d x-a^{2} \int_{0}^{T} \psi(l, t) \Delta u_{x}(l, t) d t+
\end{array}
$$




$$
\begin{aligned}
& +a^{2} \int_{0}^{T} \psi(0, t) \Delta u_{x}(0, t) d t+a^{2} \int_{0}^{T} \psi_{x}(l, t) \Delta u(l, t) d t-a^{2} \int_{0}^{T} \psi_{x}(0, t) \Delta u(0, t) d t- \\
& -\int_{0}^{T} \int_{0}^{l}\left(\psi_{t}(x, t)+a^{2} \psi_{x x}(x, t)-\lambda_{0} \psi(x, t)\right) \Delta u(x, t) d x d t- \\
& -\sum_{i=1}^{N_{s}} \sum_{j=1}^{N_{c}} \Delta \alpha_{i}^{j}\left\{\int_{0}^{T}\left[\int_{z^{i}(t)-\sigma^{\mathrm{s}}}^{z^{i}(t)+\sigma^{\mathrm{s}}} \psi(x, t) \mu_{\sigma^{\mathrm{s}}}\left(x ; z^{i}(t)\right) d x+2 \mathcal{R} g_{+}^{i}(t ; y) \operatorname{sgn}\left(g_{0}^{i}(t ; y)\right)\right]\right. \\
& \left.\left[\int_{\xi^{j}-\sigma^{\mathrm{c}}}^{\xi^{j}+\sigma^{\mathrm{c}}} u(\gamma, t) \nu_{\sigma^{\mathrm{c}}}\left(\gamma ; \xi^{j}\right) d \gamma\right] d t+2 \varepsilon\left(\alpha_{i}^{j}-\hat{\alpha}_{i}^{j}\right)\right\}+ \\
& +\sum_{i=1}^{N_{s}} \Delta \omega^{i}\left\{\int_{0}^{T}\left[\int_{z^{i}(t)-\sigma^{\mathrm{s}}}^{z^{i}(t)+\sigma^{\mathrm{s}}} \psi(x, t) \mu_{\sigma^{\mathrm{s}}}\left(x ; z^{i}(t)\right) d x+2 \mathcal{R} g_{+}^{i}(t ; y) \operatorname{sgn}\left(g_{0}^{i}(t ; y)\right)\right] d t+\right. \\
& \left.+2 \varepsilon\left(\omega^{i}-\hat{\omega}^{i}\right)\right\}-
\end{aligned}
$$$$
-\sum_{j=1}^{N_{c}} \Delta \xi^{j}\left\{\sum_{i=1}^{N_{s}} \int_{0}^{T}\left[\int_{z^{i}(t)-\sigma^{\mathrm{s}}}^{z^{i}(t)+\sigma^{\mathrm{s}}} \psi(x, t) \mu_{\sigma^{\mathrm{s}}}\left(x ; z^{i}(t)\right) d x+2 \mathcal{R} g_{+}^{i}(t ; y) \operatorname{sgn}\left(g_{0}^{i}(t ; y)\right)\right]\right.
$$$$
\left.\cdot\left[\int_{\xi^{j}-\sigma^{\mathrm{c}}}^{\xi^{j}+\sigma^{\mathrm{c}}} u_{\gamma}(\gamma, t) \nu_{\sigma^{\mathrm{c}}}\left(\gamma ; \xi^{j}\right) d \gamma\right] d t+2 \varepsilon\left(\xi^{j}-\hat{\xi}^{j}\right)\right\}-
$$$$
-\sum_{i=1}^{N_{s}} \sum_{j=1}^{N_{c}}\left\{\int_{0}^{T}\left[\int_{z^{i}(t)-\sigma^{\mathrm{s}}}^{z^{i}(t)+\sigma^{\mathrm{s}}} \psi(x, t) \mu_{\sigma^{\mathrm{s}}}\left(x ; z^{i}(t)\right) d x+2 \mathcal{R} g_{+}^{i}(t ; y) \operatorname{sgn}\left(g_{0}^{i}(t ; y)\right)\right]\right. \text {. }
$$$$
\left.\left[\int_{\xi^{j}-\sigma^{\mathrm{c}}}^{\xi^{j}+\sigma^{\mathrm{c}}} \Delta u(\gamma, t) \nu_{\sigma^{\mathrm{c}}}\left(\gamma ; \xi^{j}\right) d \gamma\right] d t\right\}
$$

It is known [19] that the components of the functional gradient are determined from (3.20) by the linear parts of its increment with respect to each of the components. Hence we have formulas (3.3)-(3.5) and the conjugate problem (3.6)-(3.8). The theorem is proved.

For discrete in time feedback (2.11), the theorem is valid. 
Theorem 3.2. Functional $J_{T, \mathcal{R}}(y)$ with discrete time feedback at each value of the penalty coefficient $\mathcal{R}$ differentiable by parameters $y=(\alpha, \omega, \xi)$, and for the components of its gradient the following formulas hold:

$$
\begin{array}{r}
\frac{\partial J_{T, \mathcal{R}}(y)}{\partial \alpha_{i}^{j}}=\int_{B} \int_{\Theta}\left\{-\sum_{k=0}^{N_{t}} \int_{t_{k}}^{t_{k+1}}\left[\int_{z^{i}(t)-\sigma^{\mathrm{s}}}^{z^{i}(t)+\sigma^{\mathrm{s}}} \psi(x, t) \mu_{\sigma^{\mathrm{s}}}\left(x ; z^{i}(t)\right) d x+\right.\right. \\
\left.+2 \mathcal{R} g_{+}^{i}\left(t_{k} ; y\right) \operatorname{sgn}\left(g_{0}^{i}\left(t_{k} ; y\right)\right)\right]\left[\int_{\xi^{j}-\sigma^{\mathrm{c}}}^{\xi^{j}+\sigma^{\mathrm{c}}} u\left(\gamma, t_{k}\right) \nu_{\sigma^{\mathrm{c}}}\left(\gamma ; \xi^{j}\right) d \gamma\right] d t+ \\
\left.+2 \varepsilon\left(\alpha_{i}^{j}-\hat{\alpha}_{i}^{j}\right)\right\} \rho_{B}(b) \rho_{\Theta}(\theta) d \theta d b,
\end{array}
$$

$$
\begin{aligned}
\frac{\partial J_{T, \mathcal{R}}(y)}{\partial \omega^{i}}= & \int_{B} \int_{\Theta}\left\{\sum _ { k = 0 } ^ { N _ { t } } \int _ { t _ { k } } ^ { t _ { k + 1 } } \left[\int_{z^{i}(t)-\sigma^{\mathrm{s}}}^{z^{i}(t)+\sigma^{\mathrm{s}}} \psi(x, t) \mu_{\sigma^{\mathrm{s}}}\left(x ; z^{i}(t)\right) d x+\right.\right. \\
& \left.\left.+2 \mathcal{R} g_{+}^{i}\left(t_{k} ; y\right) \operatorname{sgn}\left(g_{0}^{i}\left(t_{k} ; y\right)\right)\right] d t+2 \varepsilon\left(\omega^{i}-\hat{\omega}^{i}\right)\right\} \rho_{B}(b) \rho_{\Theta}(\theta) d \theta d b,
\end{aligned}
$$

$$
\begin{array}{r}
\frac{\partial J_{T, \mathcal{R}}(y)}{\partial \xi^{j}}=\int_{B} \int_{\Theta}\left\{-\sum_{i=1}^{N_{s}} \sum_{k=0}^{N_{t}} \int_{t_{k}}^{t_{k+1}}\left[\int_{z^{i}(t)-\sigma^{\mathrm{s}}}^{z^{i}(t)+\sigma^{\mathrm{s}}} \psi(x, t) \mu_{\sigma^{\mathrm{s}}}\left(x ; z^{i}(t)\right) d x+\right.\right. \\
\left.+2 \mathcal{R} g_{+}^{i}\left(t_{k} ; y\right) \operatorname{sgn}\left(g_{0}^{i}\left(t_{k} ; y\right)\right)\right]\left[\int_{\xi^{j}-\sigma^{\mathrm{c}}}^{\xi^{j}+\sigma^{\mathrm{c}}} u_{\gamma}\left(\gamma, t_{k}\right) \nu_{\sigma^{\mathrm{c}}}\left(\gamma ; \xi^{j}\right) d \gamma\right] d t+ \\
\left.+2 \varepsilon\left(\xi^{j}-\hat{\xi}^{j}\right)\right\} \rho_{B}(b) \rho_{\Theta}(\theta) d \theta d b
\end{array}
$$

$i=1, \ldots, N_{s}, j=1, \ldots, N_{c}$. Function $\psi(x, t)=\psi(x, t ; y, b, \theta, \mathcal{R})$ with the current vector of parameters $y$, admissible initial conditions $b \in B$, ambient temperature $\theta \in \Theta$ and the penalty coefficient $\mathcal{R}$ is a solution to the following conjugate initial-boundary value problem:

$$
\begin{gathered}
\psi_{t}(x, t)=-a^{2} \psi_{x x}(x, t)+\lambda_{0} \psi(x, t)-\sum_{j=1}^{N_{c}} \nu_{\sigma^{\mathrm{c}}}\left(x ; \xi^{j}\right) . \\
\sum_{i=1}^{N_{s}}\left\{\alpha_{i}^{j} \int_{z^{i}\left(t_{k}\right)-\sigma^{\mathrm{s}}}^{z^{i}\left(t_{k}\right)+\sigma^{\mathrm{s}}} \psi\left(\gamma, t_{k}\right) \mu_{\sigma^{s}}\left(\gamma ; z^{i}\left(t_{k}\right)\right) d \gamma+2 \mathcal{R} g_{+}^{i}\left(t_{k} ; y\right) \operatorname{sgn}\left(g_{0}^{i}\left(t_{k} ; y\right)\right)\right\} d t \\
x \in(0, l), t \in\left[t_{k}, t_{k+1}\right), k=0, \ldots, N_{t}, \\
\psi(x, T)=-2 \zeta(x)[u(x, T)-U(x)], x \in[0, l], \\
\psi_{x}(0, t)=\lambda \psi(0, t), \psi_{x}(l, t)=-\lambda \psi(l, t), t \in[0, T) .
\end{gathered}
$$




\section{Conclusion}

The article investigates the problem of synthesis of power control of moving sources when heating a rod. The current values of the power of the source are distributed in the neighbourhood of the points of their location and the moments of time of influence. To synthesize the power values of the sources, their dependence on the rod temperature values measured at the measurement points is constructed. At the same time, the locations of the measurement points are also optimized in the problem. Formulas are proposed for the components of the gradient in terms of the feedback parameters and the coordinates of the measurement points. The formulas obtained will make it possible to apply effective first-order optimization methods for the numerical solution of the problem.

The proposed approach can be easily extended to problems of optimal control of lumped sources described by other types of initial-boundary value problems.

\section{References}

[1] V.M. Abdullaev, K.R. Aida-zade, Numerical method of solution to loaded nonlocal boundary value problems for ordinary differential equations, Comput. Math. and Math. Phys. 54 (2014), 1096-1109. https://doi.org/10.1134/S0965542514070021

[2] V.M. Abdullaev, K.R. Aida-zade, On the numerical solution of loaded systems of ordinary differential equations, Comput. Math. and Math. Phys. 44 (2004), no. 9, $1505-1515$.

[3] V.M. Abdullayev, Numerical solution to optimal control problems with multipoint and integral conditions, Proc. Inst. Math. Mech. Natl. Acad. Sci. Azerb. 44 (2018), no. $2,171-186$.

[4] K.R. Aida-zade, V.M. Abdullaev, On an approach to designing control of the distributed parameter Processes, Autom. Remote Control. 73 (2012), no. 9, 1443-1455. https://doi.org/10.1134/S0005117912090019

[5] K.R. Aida-zade, V.A. Hashimov, Feedback control of locally lumped stabilizers for damping membrane oscillations with optimization of points of stabilizers placement and points of measuring, Journal of Modern Technology and Engineering 5 (2020), no. $2,111-128$.

[6] A.A. Alikhanov, A.M. Berezgov, M.X. Shkhanukov-Lafishev, Boundary value problems for certain classes of loaded differential equations and solving them by finite difference methods, Comput. Math. and Math. Phys. 48 (2008), 1581-1590. https://doi.org/10.1134/S096554250809008X

[7] A.G. Butkovskii, L.M. Pustyl'nikov, Teoriya podvizhnogo upravleniya sistemami s raspredelennymi parametrami (Theory of Movable Control for Systems with Distributed Parameters) (Russian), Nauka, Moscow, 1980.

[8] A.G. Butkovskii, Metody upravleniya sistemami s raspredelennymi parametrami (Control Methods for Systems with Distributed Parameters) (Russian), Nauka, Moscow, 1984.

[9] V.S. Deineka, I.V. Sergienko, Optimal'noye upravleniye neodnorodnimi raspredelennimi sistemami (Optimal control of heterogeneous distributed systems) (Russian), Naukova Dumka, Kiev, 2003.

[10] A.I. Egorov, Osnovy teorii upravleniya (Fundamentals of Control Theory) (Russian), Fizmatlit, Moscow, 2004. 
[11] S.Z. Guliyev, Synthesis of zonal controls for a problem of heating with delay under nonseparated boundary conditions, Cybern. Syst. Analysis. 54 (2018), no.1, 110121. https://doi.org/10.1007/s10559-018-0012-5

[12] M.J. Mardanov, Y.A. Sharifov, M.F. Zeynalli, Existence and uniqueness of the solutions to impulsive nonlinear integro-differential equations with nonlocal boundary conditions, Proc. Inst. Math. Mech. Natl. Acad. Sci. Azerb. 45 (2019), no. 2, 222233.

[13] A.M. Nakhushev, Nagruzhennye uravneniya i ikh prilozheniya (Loaded Equations and Their Applications) (Russian), Nauka, Moscow, 2012.

[14] B.T. Polyak, M.V. Khlebnikov, L.B. Rapoport, Mathematical Theory of Automatic Control (Russian), LENAND, Moscow, 2019.

[15] W.H. Ray, Advanced Process Control, McGraw-Hill Book Company, 1980.

[16] I.V. Sergienko, V.S. Deineka, Optimal control of distributed systems with conjugation conditions, Kluwer Acad. Publ., New York, 2005.

[17] T.K. Sirazetdinov, Optimizatsiya sistem s raspredelennymi parametrami (Optimization of Systems with Distributed Parameters) (Russian), Nauka, Moscow, 1977.

[18] V.I. Utkin, Skol'zjashhie rezhimy v zadachah optimizacii i upravlenija (Sliding Modes in Optimization and Control Problems) (Russian), Nauka, Moscow, 1981.

[19] F.P. Vasil'ev, Metody optimizatsii (Optimization Methods) (Russian), Faktorial Press, Moscow, 2002.

Kamil R. Aida-zade

Institute of Control Systems, NAS of Azerbaijan, Baku, AZ 1141, Azerbaijan, Institute of Mathematics and Mechanics, NAS of Azerbaijan, Baku, AZ 1141, Azerbaijan

E-mail address: kamil_aydazade@rambler.ru

Vugar A. Hashimov

Institute of Control Systems, NAS of Azerbaijan, Baku, AZ 1141, Azerbaijan E-mail address: vugarhashimov@gmail.com

Arzu H. Bagirov

Institute of Control Systems, NAS of Azerbaijan, Baku, AZ 1141, Azerbaijan

E-mail address: arzu-bagirov@mail.ru

Received: November 16, 2020; Accepted: March 29, 2021 